\title{
Una caracterización inicial para el logro académico de estudiantes de primer año universitario
}

\author{
First year high school student's academic achievement: \\ a preliminary picture
}

ISSN 1510-2432 - ISSN 1688-9304 (en línea) - DOI: http://dx.doi.org/10.18861/cied.2016.7.1.2575

Beatriz Carreño

Magíster en Psicología Educacional, Universidad Católica de Chile. Psicóloga, Universidad de Santiago de Chile. Investigadora en caracterización de estudiantes, impacto de programas de acompañamiento académico y estudios psicométricos relativos al rendimiento académico en la educación superior. Sub-directora, Instrumento de Caracterización Académica Inicial, Instituto CREAR, Universidad San Sebastián, Chile.

Sonia Micin

Magíster en Psicología Clínica, Universidad Católica de Chile, Ingeniera Comercial, Universidad de Santiago de Chile, Psicóloga, Universidad de Tarapacá. Investigadora en políticas educativas en educación superior, programas de acompañamiento académico en educación superior, prevalencias de salud mental en estudiantes universitarios y gestión institucional para la permanencia. Directora Nacional, Instituto CREAR, Universidad San Sebastián, Chile.

Sergio Urzua

Magíster en Sociología, Universidad Alberto Hurtado, Chile. Profesor de Estado en Filosofía, Universidad de Santiago de Chile. Investigador en políticas de equidad en educación superior, evaluación de programas institucionales para el acceso, la retención y la integración académica y social a la educación superior, trayectorias, y exclusión educativa. Metodólogo, Instituto CREAR, Universidad San Sebastián, Chile.

Fecha de recibido: 03/11/2015

Fecha de aceptado: 11/04/2016

\section{Resumen}

Las transformaciones que han operado durante las últimas décadas en la educación superior, exigen de las instituciones el compromiso de acompañar a aquellos estudiantes que por sus condiciones de ingreso pueden presentar mayores dificultades en su integración académica y social. Para esto, se requiere conocer sus necesidades y potencialidades a fin de adecuar estrategias y recursos de aprendizaje y hacer viable su experiencia académica. Desde el 2012, el Instituto CREAR de la Universidad San Sebastián, ha desarrollado un Instrumento de Caracterización Académica Inicial (ICAI) que evalúa a los estudiantes que ingresan a primer año en la Universidad. Tras cuatro años de aplicación (2012-2015), ICAI ha logrado reunir datos de más de 20.000 estudiantes. Así, este instrumento, compuesto por un cuestionario sociodemográfico, un test de habilidades cognitivas y un cuestionario de estrategias de estudio y aprendizaje, ha permitido contar con información oportuna y de calidad acerca del perfil de entrada de los estudiantes y retroalimentar las iniciativas educativas que se implementan a lo largo del proceso académico, a la vez de orientar intervenciones dirigidas a los estudiantes con mayores necesidades de apoyo. Se estima que el presente trabajo contribuye a reforzar el conocimiento responsable de las características iniciales de los estudiantes, de modo de hacer viable y exitoso su tránsito al primer año universitario. 
Palabras claves: Educación Superior, Evaluación Institucional, Características de los estudiantes, Necesidades de los estudiantes, Servicios de Apoyo Académico.

\begin{abstract}
The changes that have taken place in higher education during recent decades demand a strong commitment from educational institutions in order to provide support to students who, due to their entrance conditions, may have more difficulties in their academic and social integration. In favor to achieve this, the institutions need to know the student's needs and potentials in order to adjust strategies and learning resources to make possible their academic experience. Since 2012, the CREAR-USS Institute has developed the Initial Academic Characterization Instrument (IACI), which consists in a demographic questionnaire, a cognitive skills test and a study and learning strategies questionnaire. This instrument is applied to all freshman year college students, and after its fourth implementation (2012-2015) it has gathered data of more than 20,000 students. This information has provided valuable material regarding the characteristics of these students and has allowed necessary feedback for the academic interventions that take place during the year, drawing guidelines to develop interventions for the students that need more academic support. The following paper contributes to the importance of having valuable knowledge regarding the student's initial characteristics, in order to have a viable and successful first year in college.
\end{abstract}

Key words: Higher Education, Institutional Evaluation, Student Characteristics, Student Needs, Academic Support Services.

\title{
1. Introducción
}

En 1990 la matrícula de la Educación Superior (ES) ascendía a 245.408 estudiantes, representando el 14,2\% de la población de 18 a 24 años (Espinoza y González, 2015). Según datos del Consejo Nacional de Educación (CNED) de Chile, para el 2015 más de 1.152.000 estudiantes participaron del sistema, alcanzando una cobertura bruta del $60 \%$. Este vertiginoso aumento estaría asociado a políticas de financiamiento que permitieron el ingreso de sectores que históricamente habían sido excluidos de este nivel educativo (SIES, 2014; OCDE, 2009; Donoso, 2009). Esto último, sumado al carácter aspiracional que tiene la educación superior para la familia chilena (Fukushi, 2010), que visualiza en ella oportunidades de movilidad social y mejora de la calidad de vida, constituyen factores explicativos de la expansión y diversificación de la matrícula durante los últimos 25 años. Este proceso de masificación incorporó un importante número de estudiantes provenientes de los quintiles de menores ingresos (Brunner, 2015), que según la literatura especializada presentarían una preparación preuniversitaria insuficiente para enfrentar las nuevas demandas académicas (González, 2015; Ezcurra, 2011).

Existe amplia evidencia sobre las dificultades que enfrentan los jóvenes que provienen de contextos desfavorecidos para permanecer en la ES (Leyton, Vásquez y Fuenzalida, 2012; Castillo y Cabezas, 2010; Canales y De los Ríos, 2009; Ezcurra, 2005). Esto se manifiesta directamente en las altas tasas de deserción, las cuales se evidencian con mayor fuerza durante el primer año académico. Según datos del SIES (2014) el 30\% de los estudiantes que ingresa a la ES, la abandona al cabo del primer año, cifra que aumenta a 50\% cuando se analiza la deserción de quienes provienen de los dos primeros quintiles de ingreso (Donoso, Donoso y Frites, 2013).

Bajo este contexto, la integración académica y social de estudiantes con un nuevo perfil se ha convertido en un importante desafío para las Instituciones de Educación Superior (IES). Paulatinamente, se ha ido instalando una cultura de acompañamiento, donde el reconocimiento y el abordaje de las dificultades académicas iniciales, no constituyen 
únicamente un asunto de responsabilidad y esfuerzo personal, sino que requiere también el compromiso de las instituciones (Micin, Farías, Carreño y Urzúa, 2015; Donoso y Schiefelbein 2007; Donoso y Cancino, 2007).

A nivel general, las IES han desarrollado estrategias de retención, centradas principalmente en programas; propedéuticos, de orientación (consejería), de tutorías o mentorías, de formación docente y compensación económica (Donoso, Donoso y Frites, 2013). Sin embargo, se estima que el diseño y desarrollo de programas requiere un paso previo, que es conocer el perfil de los estudiantes que ingresan a cada institución, a saber cuáles son sus necesidades y potencialidades, de modo de gestionar aquellos soportes y apoyos académicos que sean más acordes a sus necesidades específicas y que les permitan cursar con éxito la ES.

Consistentemente con esto último, la Universidad San Sebastián (USS), en su proyecto educativo, establece como uno de los principios rectores del proceso formativo, "el respeto por el estudiante y su modo de aprender" (Proyecto Educativo-USS, 2015). Esta decisión institucional comprende una adecuación de estrategias y recursos de la enseñanza al perfil del estudiante que ingresa a la USS.

De este modo, se busca que el diseño, la implementación y la evaluación de los procesos de enseñanza y aprendizaje, se basen en el conocimiento responsable de las condiciones de ingreso de los estudiantes (Proyecto Educativo-USS, 2015).

Considerando este objetivo estratégico, el Instituto de Rendimiento y Apoyo al Estudiante (CREAR-USS), entidad que busca favorecer la integración a la vida académica universitaria, principalmente durante los primeros años universitarios, ha desarrollado desde el 2012, el Instrumento de Caracterización Académica Inicial (ICAI).

El ICAI, en tanto dispositivo de caracterización, permite contar con información relevante acerca de los estudiantes que ingresan a la Universidad, principalmente en aquellas variables asociadas al aprendizaje, la permanencia y la integración al contexto universitario. Conocer el perfil psicoeducativo de los estudiantes que ingresan a la Universidad ha permitido dirigir acciones pedagógicas, oportunas y significativas, que potencien las capacidades de los estudiantes y les brinden apoyo en sus necesidades específicas (Proyecto Educativo-USS, 2015).

\section{Descripción de ICAI: objetivos y componentes}

\subsection{Objetivos de ICAI}

El año 2015 se realizó la cuarta versión de la evaluación ICAI. Este mismo año, se consignó en el proyecto educativo de la USS, favoreciendo la socialización de sus objetivos y resultados. Este respaldo institucional también se materializa en los lineamientos entregados desde la Vicerrectoría Académica para que las diferentes carreras y unidades académicas dispongan de los recursos necesarios para el desarrollo de las aplicaciones, de modo de garantizar al menos, que el $90 \%$ de los nuevos matriculados de todas las carreras de la USS sean evaluados dentro de las dos primeras semanas de clases.

En este marco, ICAI busca responder a los siguientes objetivos institucionales:

a. Informar a las carreras USS acerca de la caracterización de sus estudiantes en cuanto a variables relevantes para el aprendizaje, el rendimiento y la adaptación al contexto universitario, y sugerir lineamientos generales de intervención.

b. Identificar estudiantes con mayor necesidad de apoyo psicoeducativo, a fin de orientar cada carrera en el diseño de metodologías educacionales pertinentes que contribuyan a la integración académica y social de este grupo.

c. Orientar los contenidos de los Programas de Acompañamiento Académico que la USS ofrece a los estudiantes nuevos (59\% de los matriculados 2015) y la selección de las carreras que participarán en ellos (50 carreras-sede de 91 totales el 2015). 
Para el logro de estos objetivos, ICAI incluye tres pruebas estandarizadas que son presentadas al estudiante a través de una plataforma web especialmente diseñada para estos efectos. La aplicación tiene un carácter presencial, una duración aproximada de una hora 20 minutos y se realiza en los laboratorios de computación de las cuatro sedes en que la Universidad tiene presencia (Santiago, Concepción, Valdivia y Puerto Montt).

La evaluación se realiza en grupos de 25 estudiantes aproximadamente y cuenta con un protocolo definido y probado, que busca controlar variables externas que pudiesen incidir en los resultados.

Por lo mismo, la evaluación es dirigida por un examinador quien es seleccionado y capacitado por el Instituto CREAR. Cada examinador cuenta con un manual de procedimiento a fin de garantizar que la aplicación sea estándar y que se respete tanto el setting de evaluación, como la progresión y tiempo de cada prueba.

\subsection{Componentes de ICAI}

El ICAI es una herramienta de evaluación compleja y multidimensional, que se compone de tres instrumentos.

a. Un Cuestionario Sociodemográfico (CSD). Este cuestionario se construyó a base de una revisión bibliográfica sobre procesos de aprendizaje, retención e integración académica en educación superior. Se recogió evidencia nacional (Canales y De los Ríos, 2009; Díaz, 2008; Donoso y Schiefelbein, 2007) e internacional (Pineda y Pedraza, 2011; Salinas \& Llanes; 2003; Corominas, 2001; Tinto, 1989; entre otras) a fin de seleccionar aquellas variables que pueden afectar con mayor fuerza la permanencia de los estudiantes. Las principales variables consideradas fueron: red de apoyo durante el periodo académico, nivel educacional de los padres, condición laboral de los padres y del estudiante, estudios anteriores, notas de enseñanza media, puntajes PSU, entre otras.

b. Un Test de Aptitudes Mentales Primarias (PMA) ${ }^{1}$. Dada la importancia de estimular las habilidades de pensamiento y el aprendizaje de los estudiantes universitarios, se ha incorporado en el ICAI el test PMA basado en las contribuciones de Thurstone (Cordero, Seisdedos, González y De la Cruz, 2007) al estudio de las habilidades cognitivas y que permite identificar en los estudiantes un perfil de su desarrollo según cuatro áreas: verbal, numérica, espacial y de razonamiento lógico.

c. Un Cuestionario de Estrategias de Estudio y Aprendizaje (CEEA) ${ }^{2}$. Este cuestionario, desarrollado por Weinstein y Palmer (2002), mide el uso que los estudiantes hacen de estrategias de aprendizaje y estudio, relacionadas con los componentes del aprendizaje estratégico (habilidad, voluntad y autorregulación). En este sentido, evalúa los pensamientos, actitudes, conductas, motivaciones y creencias desarrolladas por los estudiantes en contextos educativos anteriores y que pueden ser modificadas y mejoradas por medio de intervenciones psicoeducativas.

\section{Cobertura y resultados (2012-2015)}

\subsection{Cobertura}

ICAI pretende ser un censo de toda la cohorte que ingresa a la USS. Para identificar a los estudiantes que serán evaluados por ICAI cada año, se trabaja con la base de datos de matriculados, actualizada a principios de marzo, la que se recupera del sistema de gestión de datos institucionales Banner USS ${ }^{3}$. Se excluyen a los estudiantes que ingresan con convalidación de estudios y que por tanto, no tienen sólo cursos de primer año. Además, según la información aportada por las carreras, se omiten del análisis los estudiantes que presentan alguna situación irregular al momento de la evaluación (por ej.: abandono temprano, inasistencia, etc...). 
En la tabla 1, se observa la cobertura de ICAI para el período 2012-2015.

Tabla 1. Cobertura ICAI (2012-2015)

\begin{tabular}{c|rrrr} 
Estudiantes & $\mathbf{2 0 1 2}$ & $\mathbf{2 0 1 3}$ & $\mathbf{2 0 1 4}$ & $\mathbf{2 0 1 5}$ \\
\hline$N$ & 5.288 & 5.017 & 5.043 & 5.456 \\
$\%$ & $90,0 \%$ & $88,2 \%$ & $91,4 \%$ & $93,2 \%$
\end{tabular}

Se observa que los estudiantes evaluados por ICAI, han superado los 5.000, representando el $90 \%$ de la matrícula de cada año. Así mismo, se han evaluado el 100\% de carreras con admisión vigente en cada periodo.

\subsection{Resultados ICAI}

Tras cuatro años de aplicaciones, la información sistematizada ha permitido identificar las características de más de veinte mil estudiantes, pertenecientes a las cohortes de ingreso 2012-2015.

La tabla 2 presenta los principales resultados del cuestionario sociodemográfico.

Tabla 2. Variables contextuales y educativas periodo 2012-2015

\begin{tabular}{|c|c|c|c|c|}
\hline Variables & 2012 & 2013 & 2014 & 2015 \\
\hline $\begin{array}{l}\text { Informa vivir con alguno de } \\
\text { sus padres }\end{array}$ & $91,2 \%$ & $72,6 \%$ & $71,5 \%$ & $70,5 \%$ \\
\hline Residirá en comuna de origen & $19,8 \%$ & $26,0 \%$ & $26,6 \%$ & $26,0 \%$ \\
\hline $\begin{array}{l}\text { Trabajará el año } \\
\text { académico }\end{array}$ & $21,8 \%$ & $25,4 \%$ & $25,8 \%$ & $27,3 \%$ \\
\hline $\begin{array}{l}\text { Trabajará para financiar } \\
\text { estudios (del \% que trabaja) }\end{array}$ & $33,3 \%$ & $42,7 \%$ & $43,3 \%$ & $45,5 \%$ \\
\hline $\begin{array}{l}\text { Primera generación } \\
\text { académica }\end{array}$ & $44,4 \%$ & $52,8 \%$ & $52,2 \%$ & $50,8 \%$ \\
\hline $\begin{array}{l}\text { Alguno de los padres no ha } \\
\text { terminado la secundaria }\end{array}$ & $29,8 \%$ & $28,3 \%$ & $28,1 \%$ & $27,3 \%$ \\
\hline $\begin{array}{l}\text { Proviene de establecimientos } \\
\text { con dependencia municipal }\end{array}$ & $30,0 \%$ & $27,8 \%$ & $25,5 \%$ & $25,3 \%$ \\
\hline $\begin{array}{l}\text { Proviene de establecimientos } \\
\text { con dependencia } \\
\text { subvencionada }\end{array}$ & $50,5 \%$ & $57,6 \%$ & $60,7 \%$ & $62,1 \%$ \\
\hline $\begin{array}{l}\text { Proviene de establecimientos } \\
\text { técnico-profesionales }\end{array}$ & -- & $14,0 \%$ & $14,5 \%$ & $13,9 \%$ \\
\hline
\end{tabular}

Se observa que en los últimos tres años, el porcentaje de estudiantes de primera generación supera el $50 \%$ y más de un cuarto de los estudiantes de la USS reporta que al menos uno de sus padres no ha finalizado la educación secundaria.

También es posible informar que, de manera consistente a través de los años, los estudiantes provienen en su mayoría de establecimientos particulares subvencionados, aumentando el porcentaje desde 51\% el 2012 a 62\% el 2015. Respecto de las variables que pueden facilitar u obstaculizar la adaptación académica, en promedio cerca del 25\% 
de estudiantes trabajará durante el año académico, porcentaje que ha ido aumentando en los 4 años considerados. Igualmente, se ha incrementado el porcentaje de estudiantes que presenta algún cambio de residencia el primer año universitario, el que va desde $20 \%$ el 2012 a $26 \%$ el 2015.

En la tabla 3, se presentan los resultados del Test de Aptitudes Mentales Primarias (PMA)4.

Tabla 3. Promedios de percentiles según habilidad cognitiva

\begin{tabular}{l|cccc} 
Habilidad & 2012 & 2013 & 2014 & 2015 \\
\hline Verbal & 36 & 34 & 36 & 42 \\
Numérica & 26 & 23 & 21 & 21 \\
Razonamiento Lógico & 54 & 51 & 51 & 52 \\
Espacial & 48 & 47 & 45 & 46
\end{tabular}

Para todos los años, se observan promedios de percentiles descendidos en habilidad verbal y numérica; y promedios de percentiles alrededor del percentil 50 en habilidades de razonamiento lógico y espacial. Esto sugiere una adecuada potencialidad cognitiva para habilidades de pensamiento abstracto, las que aparecen interferidas en aquellas habilidades mediadas en mayor grado por el contexto sociocognitivo (Verbal y Numérica). En la tabla 4 se presentan los resultados del Cuestionario de Estrategias de Estudio y Aprendizaje (CEEA) ${ }^{5}$.

Tabla 4. Porcentaje de estudiantes en nivel “Bajo” según escala CEEA.

\begin{tabular}{l|cccc} 
Escalas & 2012 & 2013 & 2014 & 2015 \\
\hline Actitud ante el estudio & $36 \%$ & $38 \%$ & $42 \%$ & $45 \%$ \\
Motivación & $36 \%$ & $38 \%$ & $41 \%$ & $41 \%$ \\
Administración del tiempo & $43 \%$ & $44 \%$ & $46 \%$ & $48 \%$ \\
Manejo de la ansiedad académica & $40 \%$ & $44 \%$ & $47 \%$ & $50 \%$ \\
Gestión de la concentración & $45 \%$ & $50 \%$ & $53 \%$ & $56 \%$ \\
Procesamiento de la información & $38 \%$ & $41 \%$ & $42 \%$ & $42 \%$ \\
Selección de ideas principales & $41 \%$ & $47 \%$ & $49 \%$ & $53 \%$ \\
Ayudas de estudio & $39 \%$ & $42 \%$ & $42 \%$ & $43 \%$ \\
Autoevaluación & $36 \%$ & $37 \%$ & $40 \%$ & $40 \%$ \\
\hline Preparación de exámenes & $39 \%$ & $42 \%$ & $44 \%$ & $49 \%$
\end{tabular}


Como aparece en la tabla 4, en el periodo 2012-2015, las estrategias de estudio presentan entre $36 \%$ y $56 \%$ de estudiantes con niveles de desarrollo bajos en dichas variables. El año 2015 concentra para todas las escalas, los mayores porcentajes de estudiantes en niveles bajos, siendo mayores las dificultades en las estrategias de "Gestión de la Concentración", "Selección de ideas principales" y "Manejo de la ansiedad académica".

\section{Estudiantes con mayor necesidad de apoyo psicoeducativo}

A base de los resultados ICAI de cada estudiante, el 2012 se construyó el indicador INAP, sigla que significa Índice de Necesidad de Apoyo Psicoeducativo. Este indicador integra los resultados de todas las pruebas ICAI en un solo puntaje para cada estudiante. Para ello se realizó un índice sumatorio simple corregido (rango $=0-100$ ) para las tres pruebas de ICAI (CSD, PMA y CEEA), en función de las necesidades de apoyo psicoeducativo que los estudiantes presentan. Así, los valores altos se asocian a "mayor necesidad de apoyo psicoeducativo".

Los directores de las diferentes carreras de la USS pueden solicitar formalmente y recibir el listado que identifica a los estudiantes que presentan "mayor necesidad de apoyo psicoeducativo". De esta manera, se busca relevar la importancia de la confidencialidad de esta información, la que solo puede ser utilizada con fines de apoyo estudiantil e investigación. Luego, y según solicitud de las carreras, se realizan asesorías personalizadas a sus directores y/o docentes con el fin de orientar intervenciones específicas y oportunas con este grupo de estudiantes. Así por ejemplo, se han capacitado a Directores en habilidades de entrevista para que profundicen en las escalas que aparecen descendidas e indaguen en aquellas variables asociadas a un mayor riesgo académico. Las entrevistas han permitido a las carreras monitorear a sus estudiantes y a la vez ofrecerles soportes de apoyo específicos, según las necesidades planteadas por los estudiantes.

\section{Alcances institucionales}

Los resultados de la aplicación ICAI son sistematizados y analizados por el equipo de metodólogos de CREAR-USS para la universidad en su conjunto y para las carreras, reconociendo las especificidades de cada una de ellas. Esta caracterización académica entrega productos en seis niveles:

a. Retroalimentación de programas de apoyo académico. El modelo de diagnóstico

ICAI y las intervenciones que genera el Instituto CREAR-USS, se sostienen en el modelo teórico de Aprendizaje Estratégico desarrollado por Claire Weinstein (Weinstein \& Palmer, 2002). Esta articulación permite que la información generada en ICAI aporte a la mejora constante de las intervenciones. Así, el Programa de Nivelación Académica Inicial que busca reforzar contenidos y nivelar posibles deficiencias conceptuales en una asignatura de alta reprobación y/o complejidad; ; y el Programa de Acompañamiento Continuo Anual, que entrega apoyo académico sistemático a través de Tutorías de Pares y sesiones de atención psicoeducativas; se desarrollan y orientan considerando los resultados obtenidos en ICAI por cada carrera.

Por ejemplo, la incorporación de los Programas de Nivelación y Acompañamiento en Cálculo y en Lectura y Escritura Académica, se funda en el diagnóstico deficitario observado en el factor verbal y numérico del test PMA. Además, desde un nivel más estratégico, ICAI entrega información para decidir sobre las carreras que requieren ingresar a los programas de apoyo y las características y énfasis que deben tener estos, a fin de impactar positivamente en el logro académico de los estudiantes durante su primer año en la Universidad.

b. Retroalimentación a estudiantes para la elaboración de un plan de trabajo académico. Durante el Programa de Nivelación Académica, cada estudiante recibe los resultados 
del CEEA, los cuales son trabajados en un módulo diseñado para abordar el perfil de fortalezas y oportunidades de mejora de los estudiantes, y establecer una planificación académica, basada en las estrategias específicas que muestran sus diagnósticos. Además, durante el semestre estos resultados son profundizados en sesiones de atención psicoeducativa individual, en la que se monitorea el plan de trabajo con relación al rendimiento académico de cada estudiante.

c. Informes de carrera, escuelas y Universidad. Según los lineamientos de la Vicerrectoría académica, a mediados de abril se entrega a cada directivo de Carrera y de Escuela el informe de caracterización de su respectiva población de ingreso. La generación de informes se realiza a través de una aplicación web que automatiza el proceso y que ha permitido adelantar de manera progresiva su fecha de entrega. Cada año además, se elabora un informe general de resultados ICAI para las autoridades de la universidad?

d. Asesorías a Directivos y Docentes. El proceso de asesorías consiste en reuniones de análisis en conjunto, acerca de los resultados más importantes, profundizando en el significado y alcances de cada una de sus escalas y variables. Estas reuniones se orientan a los actores relevantes del proceso educativo y cuentan con la participación de decanos, directivos y docentes de cada carrera en particular. Su principal objetivo es facilitar la comprensión de la evaluación ICAI y entregar lineamientos generales de intervención, en concordancia con el modelo teórico en que se basan las variables medidas, y las dificultades y potencialidades detectadas en los estudiantes. Las principales temáticas abordadas son: el modelo teórico ICAI, resultados a nivel de la carrera y/o Escuela en particular y análisis de lineamientos de intervención para cada una de las escalas descendidas.

e. Capacitación Docente.

- Capacitación Docentes en el modelo CREAR-USS. En el marco del Programa de Nivelación Académica Inicial, se capacita a todos los Docentes participantes, en el perfil del estudiante USS y en Estrategias de Aprendizaje. Del mismo modo, la capacitación en Estrategias de Estudio, el perfil de ingreso ICAI y el modelo de Tutorías, constituye un requisito básico para la selección y formación de los Tutores que trabajarán con los estudiantes durante el Programa de Acompañamiento Continuo.

- Capacitación en estrategias didácticas docentes. Se desarrolla como una iniciativa conjunta con la Dirección de Desarrollo de la Docencia, dependiente de la Dirección General de Pregrado de la USS. Su objetivo es entregar a los docentes información sobre el perfil de sus estudiantes y estrategias didácticas, para ser implementadas en el aula, que favorezcan los procesos de aprendizaje de sus estudiantes. Para esto, como foco central de la intervención, se seleccionaron tres escalas que aparecen significativamente descendidas en el Cuestionario de Estudio y Estrategias de Aprendizaje que se aplica en el marco de ICAI: Gestión de la Concentración, Selección de Ideas Principales y Procesamiento de la Información.

f. Orientación a cursos de Formación Integral ${ }^{8}$. Los resultados de ICAI de cada cohorte también son utilizados por los directivos para orientar la inscripción de los cursos de Formación Integral más pertinentes a las dificultades presentadas por sus estudiantes de primer año.

Junto a esto, las carreras también desarrollan sus propios proyectos y estrategias de acompañamiento académico, las que son validadas por la Vicerrectoría Académica y asesoradas, en su mayoría, por CREAR-USS en base a los resultados obtenidos en el diagnóstico de caracterización. 


\section{Conclusiones}

A modo de cierre, es posible afirmar que en estos cuatro años de aplicación, sistematización y difusión de la información generada, ICAI se ha constituido en una importante herramienta para el conocimiento de las condiciones de ingreso de los estudiantes, la generación de dispositivos de apoyo para su integración a la vida académica universitaria, y la orientación de estrategias de enseñanza para el proceso formativo.

En este sentido, el diagnóstico ICAI reconoce el perfil de ingreso de los estudiantes y participa como un valioso insumo para ir adecuando las estrategias pedagógicas a dicho perfil. Ejemplos de esto son, la generación de Programas de Nivelación y Acompañamiento Académico acordes con las necesidades de los estudiantes y las demandas de las carreras; las capacitaciones en Estrategias de Aprendizaje y en Didácticas de aula, que han permitido a los docentes conocer las estrategias que presentan mayores dificultades y las formas de potenciarlas durante las clases; las Asesorías a Directivos y Docentes, que han favorecido la comprensión del perfil de ingreso de sus estudiantes y les ha permitido identificar a aquellos que presentan mayor necesidad de apoyo psicoeducativo, a fin de monitorear y trabajar sobre su desempeño académico.

Este aporte ha sido posible gracias al amplio compromiso institucional con que cuenta esta iniciativa, no sólo a la hora de establecer políticas que institucionalicen su ejecución año tras año, sino también a la hora de movilizar a actores relevantes en el diseño y ejecución de intervenciones pertinentes a las características de los estudiantes, y que a la vez se sostengan en el tiempo.

La gran cobertura lograda ha permitido que ICAI constituya una intervención en sí misma, dado que es una iniciativa visible a gran escala y que ha difundido el perfil psicoeducativo del estudiante USS, el que más allá de sus particularidades en algunas carreras, comparte características comunes. Todas estas últimas relativas a la brecha que existe entre las habilidades requeridas para una adaptación exitosa durante los primeros años de universidad y las que presentan los estudiantes que ingresan a la educación superior.

Esta evidencia vuelve a reforzar la responsabilidad de las instituciones y la necesidad del acompañamiento a estudiantes con nuevas demandas y oportunidades de desarrollo en la ES chilena.

\section{Referencias bibliográficas}

Brunner, J. (2015). Medio siglo de transformaciones de la educación superior chilena: Un estado del arte. En A. Bernasconi (Ed.), La educación superior de Chile. Transformación, desarrollo y crisis, páginas 21-108. Santiago de Chile: Ediciones UC.

Canales, A. y De los Ríos, D. (2009). Retención de estudiantes vulnerables en la educación universitaria chilena. Revista Calidad en la Educación, 30, pp.49-83.

Castillo, J. y Cabezas, G. (2010). Caracterización de jóvenes de primera generación en la educación superior. Nuevas trayectorias hacia la equidad educativa. Revista Calidad en la Educación, 32, pp. 43-76.

Consejo Nacional de Educación (CNED), (2015). Estadísticas y bases de datos INDICES [base de datos en línea], Santiago de Chile, [Consultado el día 29 de octubre de 2015], http://www.cned.cl/public/Seccioes/Seccion/ndicesEstadisticas/indices_estadisticas.aspx

Cordero, A., Seisdedos, N., González, M. y De la Cruz, M. (2007). Manual PMA, Aptitudes Mentales Primarias (12 ${ }^{a}$ ed. Revisada y ampliada). Madrid, España: Tea Ediciones. 
Corominas, E. (2001). La transición a los estudios universitarios. Abandono o cambio en el primer año de universidad. Revista de Investigación Educativa, 19, 1, pp. 127-151.

Díaz, C. (2008). Modelo conceptual para la deserción estudiantil universitaria chilena. Revista Estudios Pedagógicos, 2, pp. 65-86.

Donoso, S. (2009). Economía política del financiamiento de los estudios universitarios en Chile (1980-2010): debate de sus fundamentos. Innovar. Revista de Ciencias Administrativas y Sociales, 19, pp. 141-156.

Donoso, S. y Cancino, V. (2007). Caracterización socioeconómica de estudiantes de educación superior. Revista Calidad en la Educación, 26, pp. 203-244.

Donoso, S., Donoso, G. y Frites, C. (2013). La experiencia chilena de retención de estudiantes en la universidad. Revista Ciencia y Cultura, 30, pp. 141.171.

Donoso, S. y Schiefelbein, E. (2007) Análisis de los modelos explicativos de retención de estudiantes en la universidad: una visión desde la desigualdad social. Revista Estudios Pedagógicos XXXIII, 1, pp. 7-27.

Espinoza, O. y González, L. (2015). Equidad en el sistema de educación superior de Chile: acceso, permanencia, desempeño y resultados. En A. Bernasconi (Ed.), La educación superior de Chile. Transformación, desarrollo y crisis, pp. 517-579. Santiago de Chile: Ediciones UC.

Ezcurra, A. (2011) Abandono estudiantil en educación superior. Hipótesis y conceptos. En: Gluz N. (Ed.). Admisión a la universidad y selectividad social. Cuando la democratización es más que un problema de "ingresos", pp. 23-62. Buenos Aires: Universidad Nacional de General de Sarmiento.

Ezcurra, A. (2005). Diagnóstico preliminar de las dificultades de los alumnos de primer ingreso a la educación superior. Revista Perfiles Educativos, 107, pp. 118-133.

Fukushi, K (2010). El Nuevo alumno, y el desafío de la meritocracia: análisis del cambio cultural en la educación superior chilena. Revista Calidad en la Educación, 33, pp. 303-316.

Leyton, D., Vásquez, A. y Fuenzalida, V. (2012). La experiencia de estudiantes de contextos vulnerables en diferentes instituciones de educación superior universitaria (IESU): Resultados de investigación. Revista Calidad en la Educación, 37, pp. 61-97.

Micin, S., Farías, N., Carreño, B. y Urzúa, S. (2015). Beca Nivelación Académica. La experiencia de una política pública aplicada en una universidad chilena. Revista Calidad en la Educación, 42, pp. 189-208.

OCDE (2009). La educación Superior en Chile. Revisión de Políticas Nacionales de Educación. [Consultado el día 15 de septiembre de 2015] http://www.opech.cl/educsuperior/politica educacion/la_es_en_chile_ocde.pdf

Pineda, C. y Pedraza, O. (2011). Persistencia y graduación: hacia un modelo de retención estudiantil para instituciones de educación superior. Bogotá, Colombia: Colciencias. Universidad de La Sabana. 
Pinto, L., Gallardo, I. y Wenk, E. (1991). Determinación de normas para el PMA de L.L. Thurstone en estudiantes de $4^{\circ}$ año de enseñanza media científico-humanista de la Región Metropolitana. Revista de Psicología, I, 2, pp. 25-42.

Proyecto educativo-USS (2015). Proyecto Educativo Universidad San Sebastián, Santiago, Chile: Vicerrectoría Académica USS.

Salinas, A. \& Llanes J. (2003). Student Attrition, Retention, and Persistence: The Case of the University of Texas Pan American. Journal of Híspanle Higher Education, 2, 1, pp. 73-97.

Servicio de Información de Educación Superior (SIES), (2014). Panorama de la educación superior en Chile. Santiago, Chile: Mineduc.

Tinto, V. (1989). Definir la deserción: una cuestión de perspectivas. Revista de Educación Superior. Distrito Federal, México, Asociación Nacional de Universidades e Instituciones de Educación Superior (ANUIES), XVIII, 3.

Weinstein, C. \& Palmer, D. (2002) User's Manual for those administering the Learning and Study Strategies Inventory (Second Edition). Clearwater: H\&H Publishing Company, Inc.

\section{(Endnotes)}

${ }^{1}$ Coeficientes de confiabilidad entre 0,73 y 0,99.

${ }^{2}$ Coeficientes de confiabilidad entre 0,73 y 0,89 .

${ }^{3}$ Plataforma de gestión académica universitaria, entrega reportes actualizados del estado de matrícula y el rendimiento académico de los estudiantes.

${ }^{4}$ Resultados expresados en promedio de percentiles, según normas chilenas (Pinto, Gallardo, Wenk, 1991).

${ }^{5}$ Resultados expresados en porcentaje de estudiantes con habilidades descendida, según normas USS.

${ }^{6}$ El Programa de Nivelación tiene un carácter intensivo, contempla 53 módulos de 1 hora 20 minutos cada uno, distribuidos en dos semanas académicas (diez días hábiles) previos al ingreso formal a clases. Durante este período, se desarrollan dos cursos; uno asociado a la disciplina de Química, Cálculo, Biología o Lectoescritura, según la carrera a la que ingresa el estudiante; y el otro, correspondiente a Estrategias de Aprendizaje y Gestión del Tiempo.

${ }^{7}$ El año 2015 por ejemplo se entregaron 90 informes por carrera, 33 informes de Escuela y un informe general. 